S. Kwok, M. Dopita, and R. Sutherland, eds.

\title{
Collimated Outflows in Planetary Nebulae
}

\author{
José Alberto López \\ Instituto de Astronomía, UNAM, campus Ensenada, Apdo Postal 877, \\ Ensenada, Baja California, 22800, México
}

\begin{abstract}
The study of collimated outflows (COFs) in planetary nebulae (PNe) has experienced vigorous activity since the last IAU symposium on PNe, held in Groningen in 1996, for the understanding of the origin and evolution of these outflows is directly linked to the mechanisms of PN formation. Collimated outflows in planetary nebulae (PNe) have diverse characteristics and are observed as bipolar, multipolar, point-symmetric and jet-like outflows. The Hubble Space Telescope has been instrumental in revealing the complex structure and pervasive presence of COFs in a large number of PNe during the last lustrum. Significant advances have also been achieved in the study of the molecular component associated with these outflows. On the theoretical side, models that incorporate the influence of binary cores and magnetic fields have gained particular strength in explaining the origin of COFs. This review attempts to summarize some of the main progresses in the study of COFs during the last years.
\end{abstract}

\section{Introduction}

The classical notion that planetary nebulae are simply formed from isotropic, dense winds that form spherical shells has long been known to be an oversimplification of their formation mechanism. We do indeed observe round nebulae, but these are not the rule. Since the early studies on PNe (e.g. Curtis 1918) it became clear that elliptical and bipolar morphologies, representing different degrees of collimation of the stellar wind, dominate among PNe. This tendency towards axisymmetry has been clearly exemplified in every attempt that has been made to classify PNe in morphological classes (e.g. Khromov \& Kohoutek 1968; Gurzadyan 1969; Balick 1987; Schwarz, Corradi \& Stanghellini 1992; Manchado et al. 1996). A fundamental step forward in the understanding of the basic formation process and subsequent development of the nebular shell of PNe came with the interacting winds model (Kwok, Purton \& Fitzgerald 1978) that provided an elegant and sound explanation to the origin and main characteristics of the nebular envelope, including the different large-scale morphologies.

The basic concept of the interacting winds model considers first the mass lost by the star in the AGB phase as a slow and dense wind being expelled at a high mass-loss rate. Once the star leaves the AGB and moves on towards higher effective temperatures the characteristics of the mass loss process gradually change, turning into a tenous and increasingly faster wind while the mass 
loss rate effectively decreases. The fast wind eventually reaches the previously ejected, slower moving wind, and this interaction leads to a momentum exchange where hydrodynamic discontinuities are formed. An outward shock compresses, heats and accelerates the slow-moving and ambient material, forming a rim, whereas an inward facing shock decelerates, heats and compresses the stellar wind, creating a hot bubble. At the boundary between the inner and outer shock, a contact discontinuity is formed. The interacting winds model considers the hydrodynamics, radiative and thermal transfer processes involved in this situation, providing a fair general representation of the main physics involved in a wind blown bubble (e.g. see also Dyson \& de Vries 1972). In addition, axisymmetric nebulae are observed to have dense toroidal clouds surrounding the central star that must be formed at the early stages of the mass-loss process in the AGB, well before the fast wind is switched-on. When an aspherical mass distribution such as an equatorial density enhancement is introduced within the hydrodynamics of the interacting winds framework, the main observed features in axisymmetric PNe, as the elliptical and bipolar nebulae, are also succesfully reproduced (e.g. Khan \& West 1985; Mellema, Eulderinck \& Icke 1991; Frank 1999). The presence of this equatorial density enhancement is thus considered a key ingredient in the large-scale collimation process of an otherwise isotropic wind. Balick (1987), for example, based his morphological classification on this concept of an equatorial density contrast and interacting winds to interpet the different cases of elliptical and bipolar nebulae in his sample.

However, for those bipolar nebulae that show a very high degree of collimation, sometimes refered as "tight" bipolars, the presence of an equatorial toroid may not be a sufficient condition for effective collimation, unless very adhoc conditions are encountered in the surrounding environment. Furthermore, during the last years it has become clear that in addition to these large-scale axisymmetric morpholgies, the structure of PNe is far more complex than had been previously anticipated (e.g. López 1997, 2000). In an increasing number of well developed PNe the presence of secondary bipolar outflows have been detected showing a high degree of collimation and in some cases reaching expansion velocities from a few hundred $\mathrm{km} \mathrm{s}^{-1}$ to even over a thousand $\mathrm{km} \mathrm{s}^{-1}$ ! These outflows can be simply bipolar or have multiple symmetric components. In very young $\mathrm{PNe}$ and proto-PNe the pervasive presence of these complex structures has been neatly revelaed by the Hubble Space telescope (e.g. see the contribution by R. Sahai in these proceedings). In these cases the influence of magnetic fields and binary cores can provide the additional ingredients for an efficient collimation (see the contributions by G. Garcia-Segura and N. Soker in these proceedings) and an explanation for the complex geometries sometimes observed in these COFs. Moreover, at the early stages of formation of a $\mathrm{PN}$ there is abundant molecular material that has also been shown to participate in this new picture of energetic, collimated outflows emerging from young planetary nebulae (e.g. Bujarrabal et al. 2001, and see the contribution by P. Cox in these proceedings).

\section{The diversity of collimated outflows in PNe}

Some of the main characteristics and problems that represent interpreting the variety of COFs that are observed in PNe are discussed in the next sections. 


\subsection{FLIERS}

Among the first recognized forms of secondary collimated outflows in PNe were the "ansae". These are symmetric pairs of knots expanding with respect to the nucleus at velocities of the order of $50 \mathrm{~km} \mathrm{~s}^{-1}$ and are usually associated with elliptical PNe. From their general characteritics as fast, low ionization, emission regions they are known as FLIERS (c.f. Balick et al. 1998). However, an understanding of the collimation mechanism and development of their physical structure and forms, sometimes as inverted "bow-shock", has remained a challenge. Different models have been explored in trying to find an explanation to the origin of FLIERS (e.g. Dopita 1997; Redman \& Dyson 1999). A current problem in finding a consitent explanation for FLIERS is that we are now able to scrutinize the structure of PNe in far greater detail than ever before and in doing this, the original concept of a FLIER has been sometimes broadened to encompass nearly any bright knot of low ionization located within the nebular shell or in its periphery (e.g. Gonçalves, Corradi \& Mampaso, 2001). Since some of these knots may simply be due to dynamical instabilities forming in the nebular rim or be part of wider recombination fronts, one must make sure that when identifying FLIERS these actually correspond to the leading, low ionization regions of collimated outflows. In a recent model by Steffen \& López (2001) the problem of collimation is overcome considering that FLIERS are formed as the result of a lack of momentum flow along the axis of a mildly bipolar stellar wind that forms a concave bow-shock structure at the apex, where an stagnation knot is subsequently formed. This deficiency in momentum flux along the axis can be produced by a binary system. The conditions in the FLIERS of M2-9 (Livio \& Soker 2001), for example, are adequate for this model to operate. Alternatively, García-Segura \& López (2000) found in their 3D MHD numerical runs that when the mass loss rate is $<10^{-8} \mathrm{M}_{\odot} \mathrm{yr}^{-1}$, magnetic confinement will form FLIERS in elliptical and bipolar nebulae since in such conditions radiative cooling becomes efficient only at the tips (the densest part) of the jets, producing visible ansae-type structures in these regions.

\subsection{Jets}

The existence of bipolar, high-velocity jets in PNe was still a puzzle just a decade ago. Jets were known to exist in objects such as quasars, active galactic nuclei, high energy binary systems and young stars. In those objects the presence of an accretion disk and magnetic acceleration and collimation of the outflows was considered to play a key role, however these were not established components of PNe. Thus, the presence of jets in PNe presented an obvious dilemma, how are they formed and how common are they in PNe? In the search for answers to these questions we have learnt a great about the morphological structure and dynamics of PNe.

The first highly collimated mass outflow with a remarkable high expansion velocity $\left(\approx 200 \mathrm{~km} \mathrm{~s}^{-1}\right)$ found in a PN was probably the bipolar jet discovered in the Eskimo nebula, NGC 2392, by Gieseking, Becker \& Solf (1985). Bipolar jets with much higher velocities have now been found emerging from many other PNe. For example, KjPn 8 (López et al. 1997) has bipolar outflows exceeding $300 \mathrm{~km} \mathrm{~s}^{-1}$. In MyCn 18, Bryce et al. (1997) found symmetric strings of knots expanding at observed radial velocities of $500 \mathrm{~km} \mathrm{~s}^{-1}$, by all means an 
unexpected result for a PN. An even more dramatic outflow was found in the young PN He 3-1475 by Riera et al. (1995) later confirmed by Borkowski \& Harrington (2001) with data from the HST/STIS, where radial velocities reach $1200 \mathrm{~km} \mathrm{~s}^{-1}$. Furthermore, in these latter cases, as with many others, symmetry in the outflows is not just bipolar but also point-symmetric.

\subsection{Point-symmetry}

Point-symmetry was introduced as a main morphological class by Stanghellini, Corradi \& Schwarz (1993) after noticing this important peculiarity in a number of PNe. Gurzadyan (1969) had previously termed these type of PNe as SP and $\mathrm{Sz}$, meaning spiral class and spiral with $\mathrm{Z}$ shape, but little attention was paid to them until Schwarz, Corradi \& Melnick (1992) published their imaging catalogue of PNe. Point-symmetry is now known to be a common characteristic encountered in COFs of PNe of diverse morphological classes, as has been pointed out by Guerrero, Vázquez \& López (1999) and now exemplified in this symposium by the large number of works related to $\mathrm{PNe}$ with point-symmetric structures. Interestingly, when observed at high spatial resolution in the near infrared and comparing with optical images, the orientation and detailed characteristics of these structures seem to be wavelength dependent in dusty, young PNe, as has been shown by Goto et al. (2002) for AFGL 2688. Further work along these lines should be helpful in understanding the development of point-symmetry in young $\mathrm{PNe}$.

\subsection{BRETs}

In a number of cases where detailed kinematic information is available, pointsymmetric structures have been related to the presence of bipolar, rotating, episodic jets or BRETs (e.g. López, Meaburn \& Palmer 1993; López, Vázquez \& Rodríguez 1995 and Toledano et al. , these proceedings). The modeling of bipolar outflows with a seemingly progressive variation in their direction have been modeled by García-Segura (1997) via MHD models including the effects of precession from a wide binary core. More recently, García-Segura \& López (2000) considered the effects of a steady misalignment of the magnetic collimation axis with respect to the bipolar wind outflow as an alternative explanation to reproduce BRET-like cases whithout invoking an actual rotation of the outflow. This model is able to reproduce both, elliptical and bipolar nebulae with point-symmetric shapes. On a different approach, Livio \& Pringle (1996) explored the effects of a warped accretion disk produced by a close binary core that through wobbling of the (magnetically launched) bipolar jets, could reproduce point-symmetric structures. Additional information is still required in these cases to discriminate between the effects of rotation, precession and tilted COFs.

\subsection{Poly-polar nebulae}

Poly-polar nebulae have also been identified with BRETs, however in these cases, instead of observing a continuous variation of the bipolar COFs, multiple bipolar mass outflows form two or more distinct groups of bipolar lobes, such as in NGC 2440 (López et al. 1998) and KjPn 8 (López et al. 2000). There are, as yet, no 
detailed models that may explain how these multiple COFs may be produced in PNe.

Jets, point-symmetry and poly-polarity develop early in the life of a PN, the Hubble Space Telescope has shown us this in recent years beyond any doubt (see e.g. Kwok, Su \& Hrivnak 1998 and Sahai \& Trauger 1998)

\section{The molecular component in COFs}

In addition to the HST images that show the early development of complex COFs, the detection of collimated molecular outflows in very young and proto$\mathrm{PNe}$ has become in recent years an increasingly important player in their study (e.g., Huggins et al. 2000). The mass, expansion velocity and geometry of the molecular outflows are now being compared with their optical counterparts. Also, data from the Infrared Space Observatory has allowed the detection of far infrared, low excitation atomic lines that also provide measurements of the kinematics and mass from gas located in the photodissociation regions of proto-PNe (e.g. Castro-Carrizo et al 2001) that seem to follow closely the parameters obtained from the molecular material. The departure of the outflows from spherical symmetry is apparent since these early stages and has aided to better understand the structural changes that occur in the critical transition time that takes place between the AGB and proto-planetary stages.

\subsection{The energy budget in COFs}

Among the interesting new results that have emerged from the studies of molecular gas in PNe are the determination of mass, scalar momentum and kinetic energy derived from the ${ }^{12} \mathrm{CO}$ and ${ }^{13} \mathrm{CO} J=1-0$ and $J=2-1$ emission lines by Bujarrabal et al. (2001) for a relatively large sample of proto-PNe. Their findings show the strong tendency towards bipolarity in the molecular outflows, the large fraction of mass contained in them (up to $\sim 1 \mathrm{M}_{\odot}$ and more importantly, the large amount of momentum $\left(10^{37}-10^{40} \mathrm{~g} \mathrm{~cm} \mathrm{~s}^{-1}\right)$ and kinetic energy $\left(10^{44}-10^{47} \mathrm{erg}\right)$ carried by these flows. From these measurements they find an excess of momentum in the fast outflows when compared to the available budget that can be supplied by radiation pressure alone. These results suggest the need for additional energy sources as those that could be provided by the release of gravitational energy by binary interactions and magnetic fields launching the COFs along axial directions.

For the case of optical COFs, only very few estimates are available for the the momentum and kinetic energy contained in these jets. For example, Gieseking et al. (1985) estimated a mass and kinetic energy for the jet in NGC 2392 of $5 \times 10^{29} \mathrm{~g}$ and $10^{44} \mathrm{erg}$ respectively. In the case of Fleming 1 , the parameters provided by Palmer et al. (1996) yield a mass of $3.7 \times 10^{31} \mathrm{~g}$, a momentum of $3 \times 10^{38} \mathrm{~g} \mathrm{~cm} \mathrm{~s}^{-1}$ and a kinetic energy of $1.3 \times 10^{45} \mathrm{erg}$. These estimates are of course strongly dependent on the adopted distance and density that determines the volume and mass that are derived. However they seem fairly coincident with the molecular results. The implication of these values of the mass and energy budget contained in the COFs of PNe within the general evolutionary scheme of the formation of PNe are of the upmost importance and 
require modeling and additional data from the molecular, neutral and ionized components of COFs.

\section{Models for axysimmetry and COFs}

Thorough reviews on binaries and MHD models are presented in these proceedings by Soker and García-Segura. Therefore, here only a brief outline of these models is given. In order to produce axysimmetry and collimated outflows, binary cores have been invoked for some time via different mechanisms. For example, Mastrodemos and Morris (1998) investigated the formation of a disk via Bondi-Hoyle type wind accretion from the AGB star over a WD or main sequence secondary, reproducing bipolar or axisymmetric nebulae in their simulations. In an alternative model, the disk would form via Roche lobe overflow of the secondary accreting over the primary star to eventually form jets (Soker \& Livio 1994). Reyes-Ruiz \& López (1999) investigated the type of secondary companions that would lead to RLOF and the formation of an accretion disk around the primary. They found that only low mass companions $\left(\mathrm{M}_{2}<0.08\right.$ $\left.M_{\odot}\right)$ i.e. brown dwarfs or massive planets, at small orbital separations $\left(<2 R_{\odot}\right)$ may form thin accretion disks as the companion desintegrates in a dynamically unstable mass-transfer process. In these cases, accretion disks capable of providing the raw material to power collimated, high-velocity jets may be formed. The actual collimation and driving of these jets still requires of a magnetic field.

To produce the wiggles observed in the point-symmetric COFs these can be originated either by a precessing or an orbital system. The spatial and temporal variations in each case are different. For example, one expects that these fluctuation will be more extended in the case of precession and may lead to point-symmetric shapes. On the other hand, orbital systems would lead to plane or mirror symmetric COFs with shorter scale fluctuations (see e.g. Mazziari \& Raga 2002).

The production of axysimmetric outflows can also be achieved via magnetized toroidal winds from moderate rotating stars (e.g. Rózyczka \& Franco 1996). MHD models work for single stars to produce COFs and as part of binary systems, i.e. MHD and binarity are not mutually exclusive alterantives (e.g., García-Segura 1997). In these cases, if the magnetic axis of a rotating star is tilted with respect to the bipolar wind outflow, the later being perpendicular to the equatorial toroid, configurations resembling point-symmetry can be formed (García-Segura \& López 2000).

An interesting recent result by Miranda et al. (2002) has been the detection of polarized water-maser emission that delineates a magnetized torus close to the core of the young PN K 3-35, a nebula with BRET-type precessing jets.

\section{Summary of ideas}

During the last years the research on COFs in PNe has both, confirmed some previously suspected patterns and also found new elements in their structure and dynamics. 
We now know that COFs as BRETs, point-symmetric or poly-polar are ubiquitous in planetary nebulae and, as previous indications had shown, they develop since the very early stages of formation of the PN.

We have also learnt that the hydrodynamics of interacting winds alone can not account for the diversity of phenomena related with the COFs. The effects of magnetic fields and binary cores are now considered to play a fundamental role in the formation of COFs and also influence the subsequent shaping of the PN shell.

Given the apparent point-symmetry and poly-polarity now recognized in the COFs of $\mathrm{PNe}$, further analysis of their length scales and temporal variations is needed to identify the mechanisms responsible for this behaviour (e.g., precession, rotation or tilted magnetic axis). Multi-wavelength studies at high spatial resolution should also be useful in this sense.

Although the range of expansion velocities in COFs are now well characterised, from the proto-PN to more advanced stages, a better knowledge of the mass, kinetic energy and luminosities contained in them is still needed. Radiation pressure alone should not be expected to develop and sustain COFs, as it is not the case in other objects with similar phenomena, such as YSO's. There is a practical difficulty in obtaining direct observations of binary cores and magnetic fields in PNe. Therefore, confronting the contributions of the ionized, atomic and molecular components in the energy budget of the COFs to the power input that the star can provide, should aid to identify the participation of the additional energy souces that generates them, most likely gravitational interactions in binary cores and magnetic confinement and acceleration.

Acknowledgments. It is a pleasure for me to thank all my collaborators and students with whom I have worked during these years on PNe. They all have helped me to "collimate" my thinking on the subject. I also thank DGAPAUNAM and CONACYT (México) for their continuos support.

\section{References}

Balick, B. 1987, AJ, 96, 671

Balick, B., Alexander, J., terzian, Y., Perinotto, M. \& Patriarchi, P. 1998, AJ, 116, 2443

Borkowski, K. J. \& Harrington, J. P. 2001, ApJ, 550, 778

Bryce, M., López, J. A., Holloway, A. J. \& Meaburn, J. 1997, ApJ, 487, L161

Bujarrabal, V., Castro-Carrizo, A., Alcolea, J. \& Sánchez Contreras, C. 2001, A\&A, 377,868

Castro-Carrizo, A., Bujarrabal, V., Fong, D., Meixener, M., Tielens, A. G. G. M., Latter, W. B. \& Barlow, M. J. 2001, A\&A, 367, 674

Curtis, H.D. 1918, Publi. Lick Obs, 13, 55

Dopita, M. A. 1997, ApJ, 485, L41

Dyson, J.E. \& de Vries, J. 1972, A\&A, 20, 233

Frank, A., 1999, New Astronomy Reviews, 43, 31

García-Segura, G. 1997, ApJ, 489, L189

García-Segura, G. \& López, J. A. 2000, ApJ, 544, 336

Gieseking, F., Becker, I. \& Solf, J. 1985, ApJ, 295, L17

Gonçalves, D. R., Corradi, R. L. M. \& Mampaso, A. 2001, ApJ, 547, 302 
Goto, M., Kobayashi, N., Terada, H. \& Tokunaga, A. T. 2002, ApJ, in press

Guerrero, M. A., Vázquez, R. \& López, J. A. 1999, AJ, 117, 967

Gurzadyan, G. A. 1969, Planetary Nebulae (Gordon \& Breach: New York)

Huggins, P.J., Forveille, T., Bachiller, R. \& Cox, P. 2000, ApJ, 544, 889

Khan, F. D. \& West, K. 1985, MNRAS, 212, 837

Khromov, G. S. \& Kohoutek, L. 1968, in IAU Symp 34, Planetary Nebulae, ed. D.E. Osterbrock \& C.R. O'Dell (Dordrecht: Reidel) 227

Kwok, S., Purton, C. R. \& Fitzgerald, P. M. 1978, ApJ, 219, L125

Kwok, S., Su, K.-Y. \& Hrivnak, B. J., 1998, ApJ501, L117

Livio, M. \& Pringle, J. E. 1996, ApJ, 465, L55

Livio, M. \& Soker, N. 2001, ApJ, 512, 685

López, J. A., Meaburn, J. \& Palmer, J. W. 1993, ApJ, 415, L135

López, J. A., Vázquez, R. \& Rodríguez, L.F. 1995, ApJ, 455, L63

López, J. A. 1997, in IAU Symp 180, Planetary Nebulae, , ed. H. J. Habing \& H. J. G.

L. M. Lamers (Kluwer) 197

López, J. A., Meaburn, J., Bryce, M. \& Rodríguez, L.F. 1997, ApJ, 475, 705

López, J. A., Meaburn, J., Bryce, M. \& Holloway, A. J. 1998, ApJ, 493, 803

López, J. A. 2000, in Rev. Mex. Astron. Astrof. Conf. Series, Vol 9, Astrophysical Plasmas, Codes, Models and Observations, ed. J. Arthur, N. Brickhouse \& J. Franco, 201

López, J. A., Meaburn, J., Rodríguez, L.F., Steffen, W. \& Bryce, M. 2000, ApJ, 538, 233

Manchado, A., Guerrero, M. A., Stanghellini, L. \& Sierra-Ricat 1996, The IAC Morohological Catalog of Northern Galactic Planetary Nebulae, ed. Instituo de Astrofísica de Canarias

Mastrodemos, N. \& Morris, M. 1998, ApJ, 497, 303

Mazziari, E. \& Raga, A. C. 2002, ApJ, in press

Mellema, G., Eulderinck, F. \& Icke, V. 1991, A\&A, 252, 718

Miranda, L.F., Gómez, Y., Anglada, G. \& Torrelles, J. M. 2001, Nature, 414, 284

Palmer, J. W., López, J.A., Meaburn, J. \& Lloyd, H. M. 1996, A\&A, 307, 225

Redman, M. P. \& Dyson, J. E. 1999, MNRAS, 302, L17

Reyes-Ruiz, M. \& López, J. A. 1999, ApJ, 524, 952

Riera, A., García-Lario, P., Manchado, A. Pottasch, S. R. \& Raga, A. C. 1995, A\&A, 302,137

Rózyzcka M. \& Franco, J. 1996, ApJ, 469, L127

Sahai, R. \& Trauger, J. T. 1998, ApJ, 116, 1357

Schwarz, H. E., Corradi, R. L. M. \& Melnick, J. 1992, A\&A, 96, 23

Schwarz, H. E., Corradi, R. L. M. \& Stanghellini, L. 1992, in IAU Symp 155, Planetary Nebulae, ed. R. Weinberger \& A. Acker (Dordrecht: Kluwer) 214

Soker, N. \& Livio, M. 1994, ApJ, 421, 219

Stanghellini, L., Corradi, R. L. M. \& Shwarz, H. E. 1993, A\&A, 279, 521

Steffen, W. \& López, J. A. 2001 ApJ, 556, 823 